\title{
Therapeutic decision based on molecular detection of resistance mechanism in an ALK- rearranged lung cancer patient: a case report
}

\author{
Elisa De Carlol,* \\ Monica Schiappacassi $i^{2, *}$ \\ Martina Urbani ${ }^{3}$ \\ Roberto Doliana ${ }^{2}$ \\ Gustavo Baldassarre ${ }^{2}$ \\ Valentina Da Ros' \\ Sandra Santarossa' \\ Emanuela Chimienti ${ }^{\prime}$ \\ Eleonora Berto' \\ Lucia Fratino' \\ Alessandra Bearz' \\ 'Clinical Oncology Department, \\ IRCCS CRO Centro di Riferimento \\ Oncologico Aviano, Aviano, Italy; \\ 2 Molecular Oncology Department, \\ IRCCS CRO Centro di Riferimento \\ Oncologico Aviano, Aviano, Italy; \\ ${ }^{3}$ Radiology Department, IRCCS CRO \\ Centro di Riferimento Oncologico \\ Aviano, Aviano, Italy \\ *These authors contributed equally \\ to this work
}

This article was published in the following Dove Press journal: OncoTargets and Therapy

\begin{abstract}
Background: The use of tyrosine kinase inhibitors (TKIs) of ALK is the therapy of choice for ALK-fusion patients. Unfortunately, all patients under this kind of treatment eventually develop acquired resistance through several well-known mechanisms, such as acquisition of a secondary mutation within the kinase domain, activation of a bypass signaling pathway, or a histological change like small-cell lung cancer transformation. At the time of progression, a tissue re-biopsy may give important molecular and morphological information regarding the mechanisms driving resistance to ALK TKIs. However, this procedure is not always feasible and it may not reflect the tumor heterogeneity, and therefore gives incomplete information. To overcome these drawbacks, the analysis of circulating tumor DNA (ctDNA) isolated from plasma, the so-called liquid biopsy, is emerging as a noninvasive and useful tool for detecting resistance mutations. Secondary resistance mutations are common in second-generation TKIs resistant patients and among these, Gly1202Arg (p.G1202R) emerged as the most frequent mutation.
\end{abstract}

Case presentation: We have treated an ALK-positive lung adenocarcinoma patient with a sequential strategy of ALK TKIs. Patient follow-up was performed combining clinical, radiological, and molecular profiling. ctDNA was isolated from plasma and by means of ultra-deep next generation sequencing; we searched for secondary ALK resistance mutations on exons 21-25. ALK mutation Gly1202Arg (G1202R) was detected. We have documented consistency between plasma levels of G1202R mutation and radiological progression or improvement.

Conclusion: Liquid biopsy appears to be a promising tool to anticipate progression and to drive the therapeutic strategy based upon ALK resistance mutations.

Keywords: non-small-cell lung cancer, EML4-ALK, ALK tyrosine kinase inhibitors, G1202R resistance mutation, liquid biopsy

\section{Introduction}

The EML4-ALK rearrangement is a key driver gene that occurs in $3 \%-7 \%$ of non-small-cell lung cancer (NSCLC). ${ }^{1,2}$ Patients with EML4-ALK translocation (so-called ALK-positive patients) are sensitive to targeted treatment with ALK TKIs.

Crizotinib is the first-in-class generation tyrosine kinase inhibitor (TKI) approved. The therapeutic landscape for advanced $A L K$-positive patients has been transformed by the development of new generations of ALK TKIs, including ceritinib, alectinib, brigatinib, and ensartinib. These potent TKIs showed efficacy in both crizotinib naïve and resistant tumors but all patients treated with $A L K$ TKIs eventually develop $A L K$ dependent or $A L K$-independent resistances. $A L K$-independent genetic alterations include: activation of cellular mechanisms that lead to multidrug resistance (MDR), activation of bypass signaling pathways (eg, up-regulation of $E G F R$-, $K R A S$-signaling
Correspondence: Elisa De Carlo Clinical Oncology Department, IRCCS CRO Centro di Riferimento Oncologico Aviano, via Franco Gallini 2, 3308।

Aviano, Italy

Tel +390434659127

Email elisa.decarlo@cro.it 
pathways), epithelial-mesenchymal transition, and histological transformation. ${ }^{3-12}$ ALK-dependent resistance mechanisms include secondary mutations mainly located in the adenosine triphosphate binding pocket leading to the reactivation of the kinase activity despite the use of TKI.

Lorlatinib, a third-generation $A L K$ and $R O S-1$ inhibitor showed a strong effect against $A L K$-resistant mutations including G1202R and a high activity on brain metastasis. ${ }^{13}$

We report a case of an $A L K$-positive metastatic lung adenocarcinoma treated with multiple $A L K$ TKIs, characterized by the detection of G1202R in liquid biopsy; the mutation levels were consistent with clinical and radiological data during the treatment.

\section{Case presentation}

In January 2016, a 75-year-old, never-smoker woman has been diagnosed with EML4-ALK stage IV lung adenocarcinoma with bilateral lung disease (cT4N2M1a, TNM, version 7.0).

The presence of $E M L 4-A L K$ rearrangement was tested using immunohistochemistry (Ventana ALK [D5F3] CDx assay on a Ventana BenchMark XT automated slide-processing system; Ventana Medical Systems Inc., Oro Valley, AZ, UZA), with a positive immunoreaction in $80 \%$ of tumor cells.

As first-line treatment the patient received ceritinib $750 \mathrm{mg}$ once daily, in the ASCEND-8 clinical trial. ${ }^{14}$ The patient experienced clinical improvement and partial radiological response according to Response Evaluation Criteria in Solid Tumors version 1.1 (RECIST v1.1) ${ }^{15}$ until August 2016, when a computed tomography (CT) scan revealed lung progression, with a new left renal lesion and two new small brain nodules.

The patient was started on brigatinib $180 \mathrm{mg}$ daily, in an expanded access program, achieving an intra-/extra-cranial disease stability, before further systemic progression.

Since February 2017, patient follow-up was performed also by liquid biopsies. Circulating tumor DNA (ctDNA) was isolated from plasma and by means of ultra-deep next generation sequencing (NGS); we searched for secondary $A L K$ resistance mutations on exons 21-25. This approach, based on targeted exon sequencing allowed us the detection of a broad range of mutations. ${ }^{16}$

Nine different liquid biopsies were performed as depicted in Figure 1. In February, no mutations on $A L K$ exons 21-25 were detected, but in April, mutation on $A L K$ exon 23 p.G1202R was detected with a mutant allelic frequency (MAF) of $11 \%$, after eight months on brigatinib, while CT-scan showed stability.

CT scan in May revealed disease progression. The patient was then referred for a trial with a third-generation
ALK inhibitor, lorlatinib $100 \mathrm{mg}$ daily (ClinicalTrials.gov Identifier: B7461001). After a month and a half of treatment, a CT scan demonstrated disease stability according to RECIST v 1.1 and a ctDNA sample revealed that G1202R mutation was decreased to a MAF of $2.5 \%$.

In August, the G1202R mutation level in the plasma ctDNA increased to a MAF of $12 \%$; the data were radiologically confirmed, with a systemic progression, especially in the lungs, after four months of lorlatinib.

By ultra-deep NGS, we also tested the presence of activating mutations in alternative oncogenic drivers that could be linked to ALK TKI (lorlatinib) resistance. We explored ROS-1 (exon 36-37-38), EGFR (exons 18, 19, 20, 21), $K R A S$ (exons 2, 3, 4), and $B R A F$ (exon 15), but mutations were not detected by plasma-based ctDNA assay during lorlatinib treatment in none of the analyzed liquid biopsies (Figure 1). The patient was discontinued from the trial and started on chemotherapy with carboplatin/pemetrexed every three weeks in September 2017; the ctDNA sample, before starting chemotherapy, revealed a further increase of the G1202R mutation to a MAF of $14 \%$.

In October and November, during chemotherapy treatment, liquid biopsy was repeated and the $A L K$ p.G1202R mutation was not detected, with a radiological stability at CT scan. Moreover, no activating mutations in bypassing signaling pathways ( $E G F R, K R A S, B R A F$ ) were detected in plasmatic ctDNA extracted from this liquid biopsy. In December, the CT scan revealed thoracic progression but ALKp.G1202R was not detected in plasmatic ctDNA.

The patient underwent CT-guided transthoracic needle core biopsy. The re-biopsy tissue confirmed the histological diagnosis of lung adenocarcinoma and confirmed $A L K$ rearrangement by immune histochemistry; neither $A L K$ p.G1202R nor mutations in other signaling pathways ( $E G F R$, $K R A S, B R A F, R O S-1)$ were detected in the DNA extracted from tumor tissue. Based on the tissue and liquid biopsy results the chemotherapy was stopped and the patient started on alectinib in February; however, p.G1202R reappeared with a MAF of $2 \%$ after two months of treatment, and the patient passed away in April 2018 because of progressive dyspnea.

\section{Discussion}

In the reported case, we documented a potential association between liquid biopsy assay, clinical outcome, and response, which allowed us to choose among different $A L K$-targeted treatments.

The liquid biopsy allowed the identification of $A L K$ resistance mutation G1202R during treatment. The therapeutic 

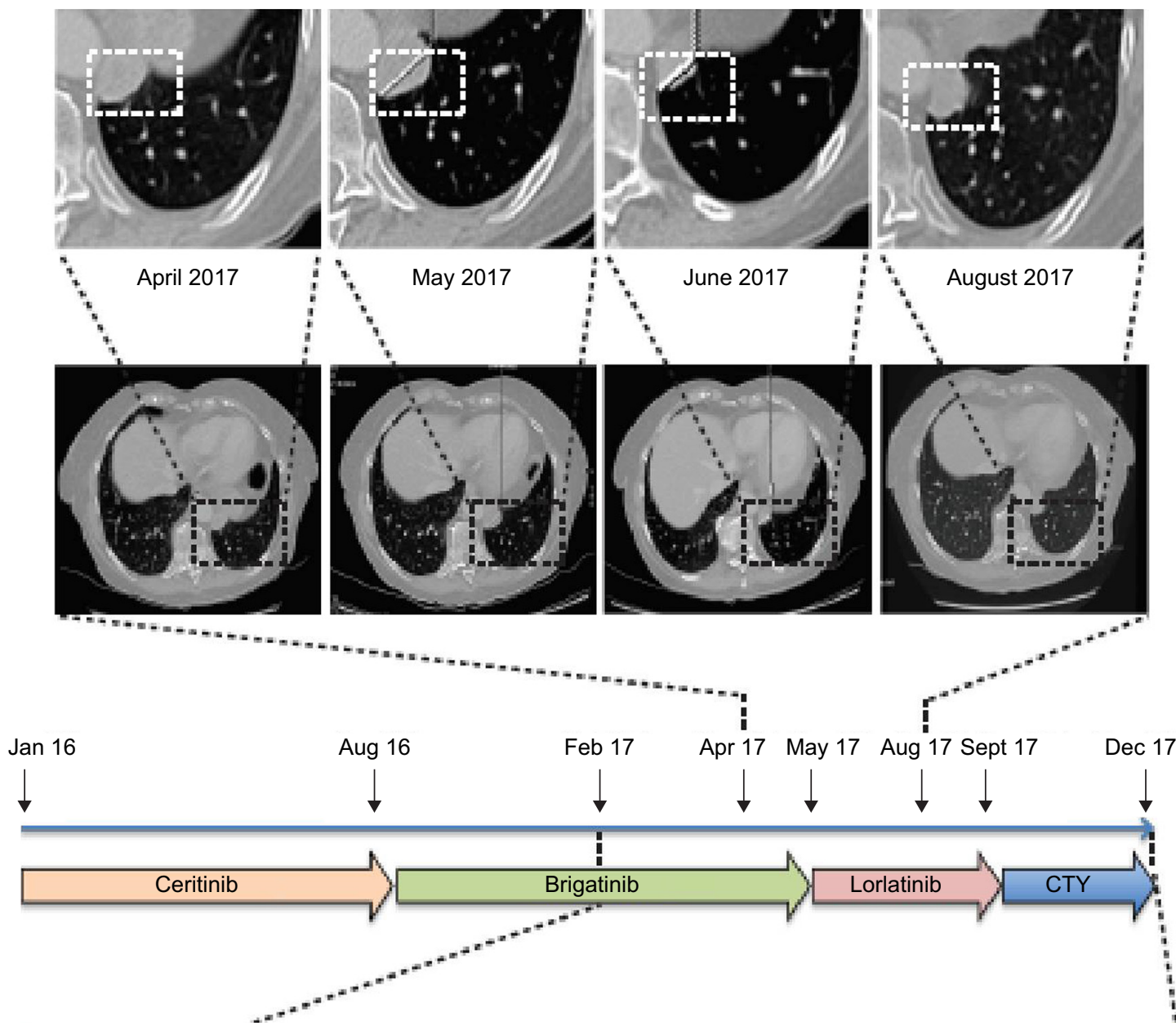

\section{Liquid biopsies 2017: ctDNA (\%MAF)}

\begin{tabular}{|c|c|c|c|c|c|c|c|c|c|}
\hline Gene & Exons & Feb & April & June & August & Sept & Oct & Nov & Dec \\
\hline ALK & $21-25$ & - & $\begin{array}{c}11 \% \\
\text { G1202R }\end{array}$ & $\begin{array}{c}2.5 \% \\
\text { G1202R }\end{array}$ & $\begin{array}{c}12 \% \\
\text { G1202R }\end{array}$ & $\begin{array}{c}14 \% \\
\text { G1202R }\end{array}$ & - & - & - \\
\hline ROS-1 & $36-38$ & - & nd & - & nd & nd & nd & nd & - \\
\hline EGFR & $18-21$ & nd & nd & - & nd & - & - & - & nd \\
\hline KRAS & $2-4$ & nd & nd & - & nd & - & - & - & nd \\
\hline BRAF & $11-15$ & nd & nd & - & nd & - & - & - & nd \\
\hline
\end{tabular}

Figure I Correlation between plasma levels of GI202R mutations detected by ctDNA assay and radiological findings.

Notes: The figure shows the sequential strategy of ALK TKIs and the time correlation between radiological findings at CT scan and the corresponding molecular profiling in liquid biopsy. In April, CT scan did not show progression of the left lower lobe mediastinal pathological tissue, however, for the first time we detected the resistance mutation GI202R in the ctDNA with II\% MAF. In May, progression was radiologically evident and for this reason, patient stopped brigatinib and entered into a clinical trial with lorlatinib. In June, CT scan demonstrated a partial remission of the left lower lobe pathological tissue, consistently with liquid biopsy, where we found a decreased amount of G I202R, 2.5\% MAF. In August, G I202R MAF increased again to I $\%$ with minor radiological progression, which was significant in September. Bypassing signaling pathways were explored in search of secondary resistance mutations, even in a tumor tissue sample, but no new activating mutations were detected and we decided to switch to chemotherapy. The white boxes show how left lower lobe mediastinal pathological tissue is increased or reduced during oncological treatments.

Abbreviations: Jan, January; Feb, February; Apr, April; Aug, August; Sept, September; Oct, October; Nov, November; Dec, December; ctDNA, circulating tumor DNA; CTY, chemotherapy; MAF, mutant allelic frequency; nd, not detected; -, negative. 
sequence has been followed and driven by G1202R plasma levels, consistent with clinical and radiological findings.

Acquired resistance to $A L K$ TKIs inevitably occurs in patients who initially respond to therapy. Gainor et al showed that resistance mutations are common in second-generation $A L K$ TKIs-resistant biopsies and G1202R emerged as the most frequent mutation. ${ }^{3}$

Lorlatinib, a third-generation $A L K$ and ROS1 inhibitor demonstrated activity against almost all $A L K$ resistance mutations, including G1202R, in preclinical models and also a high penetration in blood-brain barrier. ${ }^{17,18}$

In the Phase I trial, lorlatinb showed antitumor activity among both naïve and pretreated patients, with response also in G1202R positive tumors. ${ }^{19,20}$

The patient received brigatinib at a dose of $180 \mathrm{mg}$ per day in an expanded access program; however in the phase I trial the patients were able to receive brigatinib at a higher dose than $180 \mathrm{mg}$ per day. ${ }^{21}$ In our report, the patient was enrolled onto a trial with lorlatinib (ClinicalTrials.gov Identifier: B7461001) at the time of development of G1202R after brigatinib treatment. However, the patient showed poor treatment benefit, with radiological stability after one and a half months of therapy and both extra- and intra-cranial progression after about four months. The G1202R mutation levels in plasma ctDNA demonstrated a corresponding trend: initially a reduction at the first radiological evaluation, and then a progressive increase.

We also investigated other potential resistance mechanisms using a combination of ctDNA and tissue biopsy analyses. However, neither activation of bypassing pathways (KRAS, BRAF, EGFR) nor a histological transformation have been demonstrated, and we might likely explain the failure by a sort of pharmacokinetic resistance. Many efforts are being made to overcome MDR, among them the use of nanoparticles seems a particularly exciting field. Scientific reports described, for example, the use of siRNA coupled with nanoparticles to revert tumor MDR successfully in preclinical studies. ${ }^{10-12}$

Our case showed that tumor re-biopsy confirmed the G1202R absence, as emerged from ctDNA analysis; and the combination of the data led us to resume the target treatment with another TKI (alectinib) after chemotherapy, hoping the progression could be p.G1202R-free. Unfortunately, the aggressive p.G1202R mutation reappeared after two months on alectinib, we possibly explain the phenomenon with a temporary decrease of the clonal tumoral cells carrying p.G1202R mutation by chemotherapy, followed by re-expansion on alectinib. As we know, $A L K$ G1202R is the most frequent resistance mutation in $A L K$-rearranged lung cancer patients progressing on second-generation $A L K$ TKIs. ${ }^{3}$ To date, however, it is still a matter of debate how $A L K$ resistance mutations appear and disappear during treatment with $A L K$ TKIs, in order to suggest the optimal sequential strategy.

Our case report suggests that liquid biopsy could be a noninvasive and useful tool for detecting $A L K$ resistance mutations, similarly to what emerged in other experiences. ${ }^{22,23}$ Although tissue biopsy allows morphological information unavailable to ctDNA assay, the procedure is not always feasible. Therefore, the need to re-biopsy can be evaluated in case of discordant data between ctDNA assay and treatment response, non-informative liquid biopsy or to demonstrate a possible histological transformation. Recent studies have shown that quantitative imaging features, extracted from routine medical imaging, have prognostic value and may predict clinical outcomes or allow for treatment monitoring in different cancer types and setting. ${ }^{24-27}$ These radiomics methods in combination with machine learning or deep learning can potentially lead to better prognostic models in stage IV NSCLC, even to predict early response or progression to a treatment, to be combined with molecular profiles.

\section{Conclusions}

We have reported a case of $A L K$-positive lung adenocarcinoma treated with a sequential strategy of $A L K$ TKIs, demonstrating accordance between plasma levels of G1202R mutations detected by ctDNA assay and clinical-radiological findings.

According to our experience it is important to use liquid biopsy in the clinical practice to evaluate the efficacy of $A L K$ TKIs, to anticipate the clinical-radiological progression, and to personalize the sequential therapeutic strategy based upon $A L K$ resistance mutations. As recently stated by a multidisciplinary panel of experts on behalf of the International Association for the Study of Lung Cancer, liquid biopsy has a paramount importance in the management of advanced NSCLC with oncogene-addiction and NGS has been claimed the more appropriate method to detect the wide spectrum of $A L K$ mutations in ctDNA. ${ }^{28}$

Further prospective studies are warranted to implement a targeted therapeutic strategy through integration of clinical-radiological evaluation, pathological analysis, and liquid biopsy.

\section{Ethics approval and informed consent}

The study was performed in accordance with the Declaration of Helsinki and was approved by the ethical committee of IRCCS CRO Centro di Riferimento Oncologico, Aviano, Italy (reference number CM1702). The study participant provided written informed consent for study participation 
and data analysis, and publication of this case report and accompanying image.

\section{Availability of data and materials}

The authors have full control over the data. The data analyzed in this study are housed at the Molecular Oncology Department - IRCCS CRO Centro di Riferimento Oncologico, via Franco Gallini 2, 33081 Aviano, Italy. All relevant data are presented in the manuscript.

\section{Acknowledgment}

The authors thank Francesco Bucciotti and Sara D'Andrea for technical support.

\section{Author contributions}

Alessandra Bearz, Elisa De Carlo, and Monica Schiappacassi designed the study, wrote the manuscript, and performed the data analysis. Martina Urbani, Roberto Doliana, and Gustavo Baldassarre contributed to analyzing the data, drafting the manuscript, and scientific discussion. Valentina Da Ros, Sandra Santarossa, Emanuela Chimienti, Eleonora Berto, and Lucia Fratino helped in collecting data and taking care of the patient. All authors contributed toward data analysis, drafting and critically revising the paper, gave final approval of the version to be published, and agree to be accountable for all aspects of the work.

\section{Disclosure}

The authors report no conflicts of interest in this work.

\section{References}

1. Soda M, Choi YL, Enomoto M, et al. Identification of the transforming EML4-ALK fusion gene in non-small-cell lung cancer. Nature. 2007; 448(7153):561-566.

2. Shaw AT, Yeap BY, Mino-Kenudson M, et al. Clinical features and outcome of patients with non-small-cell lung cancer who harbor EML4-ALK. J Clin Oncol. 2009;27(26):4247-4253.

3. Gainor JF, Dardaei L, Yoda S, et al. Molecular mechanisms of resistance to first- and second-generation ALK inhibitors in ALK-rearranged lung cancer. Cancer Discov. 2016;6(10):1118-1133.

4. Ou SH, Klempner SJ, Greenbowe JR, et al. Identification of a novel HIP1ALK fusion variant in non-small-cell lung cancer (NSCLC) and discovery of ALK I1 171 (I1171N/S) mutations in two ALK-rearranged NSCLC patients with resistance to alectinib. J Thorac Oncol. 2014;9(12):1821-1825.

5. Katayama R, Shaw AT, Khan TM, et al. Mechanisms of acquired crizotinib resistance in ALK-rearranged lung cancers. Sci Transl Med. 2012; 4(120):120ra17.

6. Gainor JF, Shaw AT. Emerging paradigms in the development of resistance to tyrosine kinase inhibitors in lung cancer. J Clin Oncol. 2013; 31(31):3987-3996.

7. Miyamoto S, Ikushima S, Ono R, et al. Transformation to small-cell lung cancer as a mechanism of acquired resistance to crizotinib and alectinib. Jpn J Clin Oncol. 2016;46(2):170-173.

8. Takegawa N, Hayashi H, Iizuka N, et al. Transformation of ALK rearrangement-positive adenocarcinoma to small-cell lung cancer in association with acquired resistance to alectinib. Ann Oncol. 2016;27(5):953-955.
9. Cha YJ, Cho BC, Kim HR, Lee HJ, Shim HS. A case of ALK-rearranged adenocarcinoma with small cell carcinoma-like transformation and resistance to crizotinib. $J$ Thorac Oncol. 2016;11(5):e55-e58.

10. Liu J, Li J, Liu N, et al. In vitro studies of phospholipid-modified PAMAM-siMDR1 complexes for the reversal of multidrug resistance in human breast cancer cells. Int J Pharm. 2017;530(1-2): 291-299.

11. Guo N, Gao C, Liu J, et al. Reversal of ovarian cancer multidrug resistance by a combination of LAH4-L1-siMDR1 nanocomplexes with chemotherapeutics. Mol Pharm. 2018;15(5):1853-1861.

12. Li J, Liang H, Liu J, Wang Z. Poly (amidoamine) (PAMAM) dendrimer mediated delivery of drug and pDNA/siRNA for cancer therapy. Int $J$ Pharm. 2018;546(1-2):215-225.

13. Zou HY, Friboulet L, Kodack DP, et al. PF-06463922, an ALK/ROS1 inhibitor, overcomes resistance to first and second generation ALK inhibitors in preclinical models. Cancer Cell. 2015;28(1):70-81.

14. Cho BC, Kim DW, Bearz A, et al. ASCEND-8: A Randomized Phase 1 Study of Ceritinib, $450 \mathrm{mg}$ or $600 \mathrm{mg}$, Taken with a Low-Fat Meal versus $750 \mathrm{mg}$ in Fasted State in Patients with Anaplastic Lymphoma Kinase (ALK)-Rearranged Metastatic Non-Small Cell Lung Cancer (NSCLC). J Thorac Oncol. 2017;12(9)1357-1367.

15. Eisenhauer EA, Therasse P, Bogaerts J, et al. New response evaluation criteria in solid tumours: revised RECIST guideline (version 1.1). Eur J Cancer. 2009;45(2):228-247.

16. Bearz A, De Carlo E, Doliana R, Schiappacassi M. Acquired BRAF V600E mutation as resistant mechanism after treatment with thirdgeneration EGFR tyrosine kinase inhibitor. J Thorac Oncol. 2017; 12(11):e181-e182.

17. Zou HY, Friboulet L, Kodack DP, et al. PF-06463922, an ALK/ROS1 inhibitor, overcomes resistance to first and second generation ALK inhibitors in preclinical models. Cancer Cell. 2015;28(1):70-81.

18. Johnson TW, Richardson PF, Bailey S, et al. Discovery of (10R)-7amino-12-fluoro-2,10,16-trimethyl-15-oxo-10,15,16,17-tetrahydro$2 \mathrm{H}-8,4-($ metheno)pyrazolo[ $4,3-\mathrm{h}][2,5,11]$-benzoxadiazacyclotetr adecine-3-carbonitrile (PF-06463922), a macrocyclic inhibitor of anaplastic lymphoma kinase (ALK) and c-ros oncogene 1 (ROS1) with preclinical brain exposure and broad-spectrum potency against ALK-resistant mutations. J Med Chem. 2014;57(11): $4720-4744$.

19. Solomon BJ, Bauer TM, Felip E, et al. Safety and efficacy of lorlatinib (PF-06463922) from the dose-escalation component of a study in patients with advanced ALK+ or ROS1+ non-small cell lung cancer (NSCLC). J Clin Oncol. 2016;34(15 Suppl):9009.

20. Shaw AT, Felip E, Bauer TM, et al. Lorlatinib in non-small-cell lung cancer with ALK or ROS1 rearrangement: an international, multicentre, open-label, single-arm first-in-man phase 1 trial. Lancet Oncol. 2017; 18(12):1590-1599.

21. Gettinger SN, Bazhenova LA, Langer CJ, et al. Activity and safety of brigatinib in ALK-rearranged non-small-cell lung cancer and other malignancies: a single-arm, open-label, phase 1/2 trial. Lancet Oncol. 2016;17(12):1683-1696.

22. Bordi P, Tiseo M, Rofi E, et al. Detection of ALK and KRAS mutations in circulating tumor DNA of patients with advanced ALK-positive NSCLC with disease progression during crizotinib treatment. Clin Lung Cancer. 2017;18(6):692-697.

23. Dagogo-Jack I, Brannon AR, Ferris LA, et al. Tracking the evolution of resistance to ALK tyrosine kinase inhibitors through longitudinal analysis of circulating tumor DNA. JCO Precis Oncol. Epub 2018 Jan 23.

24. Coroller TP, Grossmann P, Hou Y, et al. CT-based radiomic signature predicts distant metastasis in lung adenocarcinoma. Radiother Oncol. 2015;114(3):345-350.

25. Fried DV, Tucker SL, Zhou S, et al. Prognostic value and reproducibility of pretreatment CT texture features in stage III non-small cell lung cancer. Int J Radiat Oncol Biol Phys. 2014;90(4):834-842.

26. Ganeshan B, Panayiotou E, Burnand K, Dizdarevic S, Miles K. Tumour heterogeneity in non-small cell lung carcinoma assessed by CT texture analysis: a potential marker of survival. Eur Radiol. 2012;22(4): 796-802. 
27. Lu CF, Hsu FT, Hsieh KL, et al. Machine learning-based radiomics for molecular subtyping of gliomas. Clin Cancer Res. 2018;24(18): 4429-4436.
28. Rolfo C, Mack PC, Scagliotti GV, et al. Liquid biopsy for advanced nonsmall cell lung cancer (NSCLC): a statement paper from the IASLC. J Thorac Oncol. 2018;13(9):1248-1268.

\section{Publish your work in this journal}

OncoTargets and Therapy is an international, peer-reviewed, open access journal focusing on the pathological basis of all cancers, potential targets for therapy and treatment protocols employed to improve the management of cancer patients. The journal also focuses on the impact of management programs and new therapeutic agents and protocols on
Dovepress

patient perspectives such as quality of life, adherence and satisfaction. The manuscript management system is completely online and includes a very quick and fair peer-review system, which is all easy to use. Visit http://www.dovepress.com/testimonials.php to read real quotes from published authors. 